\title{
New analogues of the Filbert and Lilbert matrices via products of two $k$-tuples asymmetric entries
}

\author{
Emrah Kılıç ${ }^{1}$ (D), Neşe Ömür ${ }^{2}$ (I), Sibel Koparal ${ }^{* 2}$ (C) \\ ${ }^{1}$ TOBB University of Economics and Technology, Department of Mathematics, 06560 Ankara, Turkey \\ ${ }^{2}$ Kocaeli University, Department of Mathematics, 41380 Kocaeli, Turkey
}

\begin{abstract}
In this paper, we present new analogues of the Filbert and Lilbert matrices via products of two $k$-tuples asymmetric entries consist of the Fibonacci and Lucas numbers. We shall derive explicit formulæ for their $L U$-decompositions and inverses. To prove the claimed results, we write all the identities to be proven in $q$-word and then use the celebrated Zeilberger algorithm to prove required $q$-identities.
\end{abstract}

Mathematics Subject Classification (2010). 15A23, 05A30, 11 B39

Keywords. generalized Filbert matrix, $q$-analogues, LU-decomposition, Zeilberger's algorithm, computer algebra system (CAS)

\section{Introduction}

Let $\left\{U_{n}\right\}$ and $\left\{V_{n}\right\}$ be the generalized Fibonacci and Lucas sequences, respectively, whose the Binet forms are

$$
U_{n}=\frac{\alpha^{n}-\beta^{n}}{\alpha-\beta}=\alpha^{n-1} \frac{1-q^{n}}{1-q} \text { and } V_{n}=\alpha^{n}+\beta^{n}=\alpha^{n}\left(1+q^{n}\right),
$$

where $q=\beta / \alpha=-\alpha^{-2}$, so that $\alpha=\mathbf{i} / \sqrt{q}$. When $\alpha=\frac{1+\sqrt{5}}{2}$ (or equivalently $q=$ $(1-\sqrt{5}) /(1+\sqrt{5}))$, the sequences $\left\{U_{n}, V_{n}\right\}$ are reduced to the Fibonacci sequence $\left\{F_{n}\right\}$ and the Lucas sequence $\left\{L_{n}\right\}$.

Throughout this paper we shall use the $q$-Pochhammer symbol

$$
(x ; q)_{n}=(1-x)(1-x q) \ldots\left(1-x q^{n-1}\right) .
$$

In the literature, there are many combinatorial matrices constructed by terms of special integer sequences or their functional analogues. For example, they are constructed via the binomial coefficients, the Gaussian $q$-binomial coefficients or the well-known integer sequences such as natural numbers, the Fibonacci and Lucas numbers. For these combinatorial matrices and their properties, we refer to the works $[2-6,14-17,21,22]$. Now we recall some well-known combinatorial matrices from the current literature:

\footnotetext{
*Corresponding Author.

Email addresses: ekilic@etu.edu.tr (E. Kılıç), neseomur@gmail.com (N. Ömür),

sibel.koparal@kocaeli.edu.tr (S. Koparal)

Received: 22.10.2018; Accepted: 12.03.2019
} 
- Chu and Di Claudio [5] studied the matrix $\left[\frac{(a)_{j+\lambda_{i}}}{(c)_{j+\lambda_{i}}}\right]_{0 \leq i, j \leq n}$, where $a$ and $c$ are complex numbers, $\left\{\lambda_{i}\right\}_{i=0}^{n}$ are integers and $(x)_{n}$ is the shifted factorial of order $n$. They also presented some variants of the above matrix.

- For nonnegative integer $g$, Zhou and Zhaolin [21] studied the $g$-circulant matrices whose elements consist of the Fibonacci and Lucas numbers, separately.

- Hilbert matrix $\mathcal{H}=\left[h_{i j}\right]$ is defined with entries

$$
h_{i j}=\frac{1}{i+j-1} .
$$

- As an analogue of the well known Hilbert matrix, Richardson [20] defined and studied the Filbert matrix $H_{n}=\left[\widetilde{h}_{i j}\right]_{i, j=1}^{n}$ with entries

$$
\widetilde{h}_{i j}=\frac{1}{F_{i+j-1}}
$$

where $F_{n}$ is the $n$th Fibonacci number.

- The Filbert matrix has been extended by Berg [1] and Ismail [7].

- Also several generalizations and analogues of it have been investigated and studied. For example, Kılıç and Prodinger [8] gave a generalization of the Filbert matrix denoted by $\mathcal{F}$ with entries

$$
f_{i j}=\frac{1}{F_{i+j+r}},
$$

where $r \geq-1$ is an integer parameter.

- Kllıç and Prodinger [10] introduced two new variations of the Filbert matrix $\mathcal{F}$ denoted by $\mathcal{G}$ and $\mathcal{L}$ with entries, respectively

$$
g_{i j}=\frac{F_{\lambda(i+j)+r}}{F_{\lambda(i+j)+s}} \quad \text { and } \quad l_{i j}=\frac{L_{\lambda(i+j)+r}}{L_{\lambda(i+j)+s}},
$$

where $s, r$, and $\lambda$ are integer parameters such that $s \neq r, s \geq-1$, and $\lambda \geq 1$. This was the first nontrivial example where the numerator of the entries is not equal to zero.

- Kılıç and Prodinger [9] gave a further generalization of the generalized Filbert matrix $\mathcal{F}$ by defining the matrix $Q$ with entries

$$
Q_{i j}=\frac{1}{F_{i+j+r} F_{i+j+r+1} \ldots F_{i+j+r+k-1}},
$$

where $r \geq-1$ is an integer parameter and $k \geq 0$ is an integer parameter.

- Some authors generalized and extended the concept in a series of papers $[8-13,19]$ to matrices with entries

$$
\frac{1}{F_{\lambda(i+j)+r} F_{\lambda(i+j+1)+r} \cdots F_{\lambda(i+j+k-1)+r}}
$$

and

$$
\frac{1}{L_{\lambda(i+j)+r} L_{\lambda(i+j+1)+r} \ldots L_{\lambda(i+j+k-1)+r}},
$$

where $r \geq-1$ and $\lambda, k \geq 1$ are integer parameters.

- Kılıç and Prodinger [11] went one step further, by allowing an asymmetric growth of indices. They, however, confined themselves to $k=1$; for this instance, the inverse matrix also enjoys nice closed form entries, which is no longer true for 
$k \geq 2$. To be more specific, they introduced four generalizations of the Filbert matrix $\mathcal{F}$, and defined the matrices $\mathcal{T}, \mathcal{M}, \mathcal{H}$, and $\mathcal{Z}$ with entries by

$$
t_{i j}=\frac{1}{F_{\lambda i+\mu j+r}}, m_{i j}=\frac{F_{\lambda i+\mu j+r}}{F_{\lambda i+\mu j+s}}, h_{i j}=\frac{1}{L_{\lambda i+\mu j+r}} \text { and } z_{i j}=\frac{L_{\lambda i+\mu j+r}}{L_{\lambda i+\mu j+s}},
$$

respectively, where $s, r, \lambda$, and $\mu$ are integer parameters such that $s \neq r, s, r \geq-1$, and $\lambda, \mu \geq 1$. To prove their results, the authors could not use the $q$-Zeilberger algorithm because the summand they needed, are not $q$-hypergeometric. So they used the backward induction to prove their claims.

In these summarized works, the authors derived explicit formulæ for the $L U$-decompositions for the matrices mentioned above. Also they derived explicit formulæ for their inverses.

In this paper, inspiring by the all works mentioned above, we shall present new analogues of the Filbert and Lilbert matrices. We study two matrices $A$ and $B$ with entries

$$
A_{i j}=\frac{1}{\left(F_{i+j+r+1} F_{i+j+r+2 \ldots} \ldots F_{i+j+r+k}\right) \cdot\left(L_{i-j+s+1} L_{i-j+s+2 \ldots} \ldots L_{i-j+s+k}\right)}
$$

and

$$
B_{i j}=\frac{1}{\left(L_{i+j+r+1} L_{i+j+r+2} \ldots L_{i+j+r+k}\right) \cdot\left(L_{i-j+s+1} L_{i-j+s+2} \ldots L_{i-j+s+k}\right)},
$$

where $r, s, \lambda$, and $\mu$ are integer parameters such that $s \neq r, r, s \geq-1$, and $\lambda, \mu \geq 1$. We shall derive explicit formulæ for the $L U$-decompositions and their inverses.

Thus we denote $q$-forms of the matrices $A$ and $B$ by $\mathcal{A}$ and $\mathcal{B}$, respectively. In that case, they are

$$
\begin{aligned}
\mathcal{A}_{n, d} & =(1-q)^{k}(-1)^{k(n+r+s+1)+1} \mathbf{i}^{k(k+s+r)} q^{\frac{1}{2} k(k+r+s)+n k} \\
& \times \frac{1}{\prod_{t=1}^{k}\left[\left(1-q^{n+d+r+t}\right)\left(1+q^{n-d+s+k}\right)\right]}
\end{aligned}
$$

and

$$
\begin{aligned}
\mathcal{B}_{n, d} & =(-1)^{k(n+r+s)+1} \mathbf{i}^{k(k+r+s+1)} q^{\frac{1}{2} k(r+s+k+1)+k n} \\
& \times \frac{1}{\prod_{t=1}^{k}\left[\left(1+q^{n+d+r+t}\right)\left(1+q^{n-d+s+k}\right)\right]},
\end{aligned}
$$

respectively. After this, we will present all our results for the matrices $\mathcal{A}$ and $\mathcal{B}$ because all our identities hold for a general $q$. Thus one can obtain the results related with the matrices $A$ and $B$ by taking $q=(1-\sqrt{5}) /(1+\sqrt{5})$.

We briefly clarify what will be done throughout this paper. We will derive explicit formulæ for $L U$-decompositions and inverses of the matrices $\mathcal{A}$ and $\mathcal{B}$. Here we only prove some of the claimed results rather than all of them. We will use the celebrated $q$-Zeilberger algorithm [19] to prove the claimed results. In detail, all the results related with the matrix $\mathcal{A}$ will be listed in Section 2 without proof. All the results related with the matrix $\mathcal{B}$ will be listed in Section 3 without proof. We also give Fibonacci-Lucas corollaries of our results after each result by choosing a special value of $q, q=(1-\sqrt{5}) /(1+\sqrt{5})$ in Sections 2 and 3. Then, in Section 4.1, we will give the proofs related with the matrix $\mathcal{A}$. For the matrix $\mathcal{A}$, we prove the claim about the $L U$-decompositions of the matrix $\mathcal{A}$ as well as the claim about the matrix $L$ and its inverse matrix $L^{-1}$. In Section 4.2, for the matrix $\mathcal{B}$, we prove the claim about the $L U$-decompositions of the matrix $\mathcal{B}$ as well as the claim about the matrix $U$ and its inverse matrix $U^{-1}$. While proving the claimed identities mentioned just above, we use the $q$-Zeilberger algorithm. 


\section{The main results}

In this section, we will present all our results related to matrix $\mathcal{A}$. We start with giving the matrices $L$ and $U$ yielding from the $L U$-decomposition of the matrix $\mathcal{A}$ of the form $\mathcal{A}=L \cdot U$ in the following next two theorems.

Theorem 2.1. For $1 \leq d \leq n$,

$$
\begin{aligned}
L_{n, d} & =(-q)^{k(n-d)} \frac{(q ; q)_{n-1}}{(q ; q)_{n-d}(q ; q)_{d-1}} \\
& \times \frac{\left(q^{d+r+2} ; q\right)_{d+k-1}\left(-q^{s+1} ; q\right)_{d+k-1}\left(-q^{2 d+k+s+r+1} ; q\right)_{n-d}}{\left(q^{n+r+2} ; q\right)_{d+k-1}\left(-q^{n-d+s+1} ; q\right)_{d+k-1}\left(-q^{d+k+s+r+2} ; q\right)_{n-d}} .
\end{aligned}
$$

Fibonacci-Lucas corollary for $k=3, r=4$, and $s=2$ :

Corollary 2.2. For $1 \leq d \leq n$,

$$
L_{n, d}=\frac{(-1)^{n-d}\left(\prod_{t=1}^{n-1} F_{t}\right)\left(\prod_{t=1}^{d+2} F_{t+d+5}\right)\left(\prod_{t=1}^{d+2} L_{t+2}\right)\left(\prod_{t=1}^{n-d} L_{t+2 d+9}\right)}{\left(\prod_{t=1}^{n-d} F_{t}\right)\left(\prod_{t=1}^{d-1} F_{t}\right)\left(\prod_{t=1}^{d+2} F_{t+n+5}\right)\left(\prod_{t=1}^{d+2} L_{t+n-d+2}\right)\left(\prod_{t=1}^{n-d} L_{t+d+10}\right)} .
$$

Theorem 2.3. For $1 \leq d \leq n$,

$$
\begin{aligned}
U_{d, n} & =(-1)^{k(d-1)} \mathbf{i}^{k(k-r-s)} \\
& \times q^{d+k-1-n(d-1)+\frac{k(k+r+s)}{2}}(1-q)^{k} \frac{(q ; q)_{n-1}}{(q ; q)_{n-d}} \\
& \times \frac{\left(q^{k} ; q\right)_{d-1}\left(-q^{d+k+r+s+2} ; q\right)_{d-1}\left(-q^{n+r-s+1} ; q\right)_{d-1}}{\left(-q^{-d-k-s+1} ; q\right)_{d-1}\left(q^{d+k+r+1} ; q\right)_{d-1}\left(q^{n+r+2} ; q\right)_{d+k-1}\left(-q^{s-n+2} ; q\right)_{d+k-1}} .
\end{aligned}
$$

Fibonacci-Lucas corollary for $k=3, r=4$, and $s=2$ :

Corollary 2.4. For $1 \leq d \leq n$,

$$
\begin{aligned}
U_{d, n} & =(-1)^{-n(d-1)+\frac{3}{2}} 5^{3 / 2} \\
& \times \frac{\left(\prod_{t=1}^{d-1} F_{t+2}\right)\left(\prod_{t=1}^{n-1} F_{t}\right)\left(\prod_{t=1}^{d-1} L_{t+d+10}\right)\left(\prod_{t=1}^{d-1} L_{t+n+2}\right)}{\left(\prod_{t=1}^{n-d} F_{t}\right)\left(\prod_{t=1}^{d-1} L_{t-d-5}\right)\left(\prod_{t=1}^{d-1} F_{t+d+7}\right)\left(\prod_{t=1}^{d+2} F_{t+n+5}\right)\left(\prod_{t=1}^{d+2} L_{t-n+3}\right)} .
\end{aligned}
$$

Now we shall formulate determinant of the matrix $\mathcal{A}$ in the following theorem.

Theorem 2.5. For $1 \leq n, d \leq N$,

$$
\begin{aligned}
\operatorname{det} \mathcal{A}_{N} & =(-1)^{k N} \mathbf{i}^{k(k-r-s) N} q^{\left(k-1+n+\frac{k(k+r+s)}{2}\right) N} \\
& \times(1-q)^{k N} \prod_{d=1}^{N}(-1)^{k d} q^{d(1-n)} \\
& \times \frac{\left(-q^{d+k+r+s+2} ; q\right)_{d-1}\left(q^{k} ; q\right)_{d-1}(q ; q)_{d-1}\left(-q^{d+r-s+1} ; q\right)_{d-1}}{\left(-q^{-d-k-s+1} ; q\right)_{d-1}\left(q^{d+k+r+1} ; q\right)_{d-1}\left(q^{d+r+2} ; q\right)_{d+k-1}\left(-q^{s-d+2} ; q\right)_{d+k-1}} .
\end{aligned}
$$

Now we present the inverse matrices $L^{-1}$ and $U^{-1}$ in the following next two theorems. 
Theorem 2.6. For $1 \leq d \leq n$,

$$
\begin{aligned}
L_{n, d}^{-1} & =(-1)^{(k-1)(d-n)} q^{\left(\frac{n-d+1}{2}\right)+(k-1)(n-d)} \\
& \times\left(1+q^{2 d+k+r+s+1}\right) \frac{(q ; q)_{n-1}}{(q ; q)_{n-d}(q ; q)_{d-1}} \\
& \times \frac{\left(q^{d+r+2} ; q\right)_{n-d}\left(-q^{d-n+s+2} ; q\right)_{n-d}\left(-q^{n+k+s+r+2} ; q\right)_{n-1}}{\left(q^{n+d+k+r} ; q\right)_{n-d}\left(-q^{d+k+s} ; q\right)_{n-d}\left(-q^{d+k+s+r+2} ; q\right)_{n}} .
\end{aligned}
$$

Fibonacci-Lucas corollary for $k=3, r=4$, and $s=2$ :

Corollary 2.7. For $1 \leq d \leq n$,

$$
\begin{aligned}
L_{n, d}^{-1} & =(-1)^{\left(\begin{array}{c}
d \\
2
\end{array}\right)+\left(\begin{array}{c}
n+1 \\
2
\end{array}\right)-d n} L_{2(d+5)} \\
& \times \frac{\left(\prod_{t=1}^{n-1} F_{t}\right)\left(\prod_{t=1}^{n-d} F_{t+d+5}\right)\left(\prod_{t=1}^{n-d} L_{t+d-n+3}\right)\left(\prod_{t=1}^{n-1} L_{t+n+10}\right)}{\left(\prod_{t=1}^{n-d} F_{t}\right)\left(\prod_{t=1}^{d-1} F_{t}\right)\left(\prod_{t=1}^{n-d} F_{t+n+d+6}\right)\left(\prod_{t=1}^{n-d} L_{t+d+4}\right)\left(\prod_{t=1}^{n} L_{t+d+10}\right)} .
\end{aligned}
$$

Theorem 2.8. For $1 \leq d \leq n$,

$$
\begin{aligned}
U_{d, n}^{-1} & =(-1)^{d+n(k-1)} \mathbf{i}^{k(k+r+s)} q^{\left(\begin{array}{c}
d-1 \\
2
\end{array}\right)+\left(\begin{array}{c}
n \\
2
\end{array}\right)+s-k \frac{k+r+s}{2}} \frac{\left(1+q^{2 d+r-s}\right)}{(1-q)^{k}} \\
& \times \frac{\left(q^{d+r+2} ; q\right)_{n+k-2}\left(-q^{s+2-d} ; q\right)_{n+k-2}\left(q^{n+k+r+1} ; q\right)_{n}\left(-q^{-n-k-s+1} ; q\right)_{n}}{\left(q^{k} ; q\right)_{n-1}(q ; q)_{n-d}(q ; q)_{d-1}\left(-q^{d+r-s+1} ; q\right)_{n}\left(-q^{n+k+r+s+2} ; q\right)_{n-1}} .
\end{aligned}
$$

Fibonacci-Lucas corollary for $k=3, r=4$, and $s=2$ :

Corollary 2.9. For $1 \leq d \leq n$,

$$
\begin{aligned}
U_{d, n}^{-1} & =(-1)^{\left(\begin{array}{c}
d \\
2
\end{array}\right)+\left(\begin{array}{c}
n+2 \\
2
\end{array}\right)} L_{2(d+1)} 5^{-3 / 2} \\
& \times \frac{\left(\prod_{t=1}^{n+1} F_{t+d+5}\right)\left(\prod_{t=1}^{n+1} L_{t-d+3}\right)\left(\prod_{t=1}^{n} F_{t+n+7}\right)\left(\prod_{t=1}^{n} L_{t-n-5}\right)}{\left(\prod_{t=1}^{n-1} F_{t+2}\right)\left(\prod_{t=1}^{n-d} F_{t}\right)\left(\prod_{t=1}^{d-1} F_{t}\right)\left(\prod_{t=1}^{n} L_{t+d+2}\right)\left(\prod_{t=1}^{n-1} L_{t+n+10}\right)} .
\end{aligned}
$$

Now let us consider the inverse matrix again. Since $\mathcal{A}^{-1}=U^{-1} L^{-1}$ and by the definitions of the matrices $U^{-1}$ and $L^{-1}$, we have the following result without proof.

Theorem 2.10. For $1 \leq i, j \leq n$, we have

$$
\begin{aligned}
& \left(\left(\mathcal{A}_{n}\right)^{-1}\right)_{i, j}=\mathbf{i}^{k(k+r+s)}(-1)^{j k-j+i} \\
& \times q_{2}^{(j+1)}-\frac{1}{2} k(r+s+k)+s-j k-\frac{1}{2}(3 i-1) \frac{\left(1+q^{2 j+k+r+s+1}\right)\left(1+q^{2 i+r-s}\right)}{(1-q)^{k}(q ; q)_{i-1}(q ; q)_{j-1}} \\
& \times \sum_{\max \{i, j\} \leq t \leq n} q^{t(t-j+k-1)} \frac{\left(q^{i+r+2} ; q\right)_{t+k-2}\left(-q^{s+2-i} ; q\right)_{t+k-2}\left(q^{t+k+r+1} ; q\right)_{t}}{\left(q^{k} ; q\right)_{t-1}\left(-q^{i+r-s+1} ; q\right)_{t}\left(-q^{t+k+r+s+2} ; q\right)_{t-1}} \\
& \times \frac{(q ; q)_{t-1}\left(-q^{-t-k-s+1} ; q\right)_{t}\left(q^{j+r+2} ; q\right)_{t-j}\left(-q^{j-t+s+2} ; q\right)_{t-j}\left(-q^{t+k+s+r+2} ; q\right)_{t-1}}{(q ; q)_{t-i}(q ; q)_{t-j}\left(q^{t+j+k+r} ; q\right)_{t-j}\left(-q^{j+k+s} ; q\right)_{t-j}\left(-q^{j+k+s+r+2} ; q\right)_{t}} .
\end{aligned}
$$


The final formula given in the last theorem follows from some straightforward simplifications. Unfortunately, the sum cannot be evaluated in closed form as we saw.

\section{The matrix $\mathcal{B}$}

Now we present all our results related to the matrix $\mathcal{B}$. For convenience, we use the same letters $L$ and $U$, but with a different meaning. We present the matrices $L$ and $U$ yielding from the $L U$-decomposition $\mathcal{B}=L . U$ in the following next two theorems:

Theorem 3.1. For $1 \leq d \leq n$,

$$
\begin{aligned}
L_{n, d} & =(-q)^{k(n-d)} \frac{\left(q^{n-d+1} ; q\right)_{d-1}}{(q ; q)_{d-1}} \\
& \times \frac{\left(-q^{d+r+2} ; q\right)_{d+k-1}\left(-q^{s+1} ; q\right)_{d+k-1}\left(q^{n+k+r+s+2} ; q\right)_{d-1}}{\left(-q^{n+r+2} ; q\right)_{d+k-1}\left(-q^{n-d+s+1} ; q\right)_{d+k-1}\left(q^{d+k+r+s+2} ; q\right)_{d-1}} .
\end{aligned}
$$

Fibonacci-Lucas corollary for $k=3, r=4$, and $s=2$ :

Corollary 3.2. For $1 \leq d \leq n$,

$$
\begin{aligned}
L_{n, d}= & (-1)^{n-d} \\
\times & \frac{\left(\prod_{t=1}^{d-1} F_{t+n-d}\right)\left(\prod_{t=1}^{d+2} L_{t+s}\right)\left(\prod_{t=1}^{d+2} L_{-t+d+2}\right)\left(\prod_{t=1}^{d-1} F_{t+n+10}\right)}{\left(\prod_{t=1}^{d-1} F_{t}\right)\left(\prod_{t=1}^{d+2} L_{t+n+5}\right)\left(\prod_{t=1}^{d+2} L_{t+n-d+2}\right)\left(\prod_{t=1}^{d-1} F_{t+d+10}\right)} .
\end{aligned}
$$

Theorem 3.3. For $1 \leq d \leq n$,

$$
\begin{aligned}
U_{d, n} & =(-1)^{k(d-1)} \mathbf{i}^{k(k-r-s-1)} q^{(n-1)(1-d)+k\left(\frac{r+s+2}{2}\right)+\left(\begin{array}{c}
k+1 \\
2
\end{array}\right)} \\
& \times \frac{\left(q^{n+r-s+1} ; q\right)_{d-1}}{\left(-q^{n+r+2} ; q\right)_{d+k-1}\left(-q^{-n+s+2} ; q\right)_{d+k-1}} \\
& \times \frac{\left(q^{k} ; q\right)_{d-1}(q ; q)_{n-1}\left(q^{d+k+r+s+2} ; q\right)_{d-1}}{(q ; q)_{n-d}\left(-q^{d+k+r+1} ; q\right)_{d-1}\left(-q^{-d-k-s+1} ; q\right)_{d-1}} .
\end{aligned}
$$

Fibonacci-Lucas corollary for $k=3, r=4$, and $s=2$ :

Corollary 3.4. For $1 \leq d \leq n$,

$$
\begin{aligned}
U_{d, n}= & (-1)^{n(1-d)} \\
& \times \frac{\left(\prod_{t=1}^{d-1} F_{t+2}\right)\left(\prod_{t=1}^{n-1} F_{t}\right)\left(\prod_{t=1}^{d-1} F_{t+n+2}\right)\left(\prod_{t=1}^{d-1} F_{t+d+10}\right)}{\left(\prod_{t=1}^{n-d} F_{t}\right)\left(\prod_{t=1}^{d+2} L_{t+n+5}\right)\left(\prod_{t=1}^{d-1} L_{t+d+7}\right)\left(\prod_{t=1}^{d+2} L_{t-n+3}\right)\left(\prod_{t=1}^{d-1} L_{t-d-5}\right)} .
\end{aligned}
$$

We can give determinant of the matrix $\mathcal{B}$ since it is simply evaluated as product of the main diagonal elements of the matrix $U$ yielding from the $L U$-decomposition $\mathcal{B}=L . U$ in the following theorem. 
Theorem 3.5. For $1 \leq n, d \leq N$,

$\operatorname{det} \mathcal{B}_{N}$

$$
\begin{aligned}
& =(-1)^{k N} \mathbf{i}^{k(k-r-s-1) N} q^{\left(n-1+k\left(\frac{r+s+2}{2}\right)+\left(\begin{array}{c}
k+1 \\
2
\end{array}\right)\right) N} \prod_{d=1}^{N}(-1)^{k d} q^{-d(n-1)} \\
& \times \frac{\left(q^{k} ; q\right)_{d-1}(q ; q)_{d-1}\left(q^{d+r-s+1} ; q\right)_{d-1}\left(q^{d+k+r+s+2} ; q\right)_{d-1}}{\left(-q^{d+k+r+1} ; q\right)_{d-1}\left(-q^{-d-k-s+1} ; q\right)_{d-1}\left(-q^{d+r+2} ; q\right)_{d+k-1}\left(-q^{-d+s+2} ; q\right)_{d+k-1}} .
\end{aligned}
$$

Now we present the inverse matrices $L^{-1}$ and $U^{-1}$ in the following next two theorems.

Theorem 3.6. For $1 \leq d \leq n$,

$$
\begin{aligned}
L_{n, d}^{-1} & =(-1)^{(k+1)(n-d)} q^{\left(\frac{n-d+1}{2}\right)+(k-1)(n-d)} \\
& \times\left(1-q^{2 d+r+s+k+1}\right) \frac{(q ; q)_{n-1}}{(q ; q)_{n-d}(q ; q)_{d-1}} \\
& \times \frac{\left(-q^{d+r+2} ; q\right)_{n-d}\left(-q^{d-n+s+2} ; q\right)_{n-d}\left(q^{n+k+r+s+2} ; q\right)_{n-1}}{\left(-q^{n+d+k+r} ; q\right)_{n-d}\left(-q^{d+k+s} ; q\right)_{n-d}\left(q^{d+k+r+s+2} ; q\right)_{n}} .
\end{aligned}
$$

Fibonacci-Lucas corollary for $k=3, r=4$, and $s=2$ :

Corollary 3.7. For $1 \leq d \leq n$,

$$
\begin{aligned}
L_{n, d}^{-1}= & (-1)^{\left(\begin{array}{c}
d \\
2
\end{array}\right)+\left(\begin{array}{c}
n+1 \\
2
\end{array}\right)-d n-d+1} L_{2(d+5)} \\
& \times \frac{\left(\prod_{t=1}^{n-1} F_{t}\right)\left(\prod_{t=1}^{n-d} L_{t+d+5}\right)\left(\prod_{t=1}^{n-d} L_{t+d-n+3}\right)\left(\prod_{t=1}^{n-1} F_{t+n+10}\right)}{\left(\prod_{t=1}^{n-d} F_{t}\right)\left(\prod_{t=1}^{d-1} F_{t}\right)\left(\prod_{t=1}^{n-d} L_{t+n+d+6}\right)\left(\prod_{t=1}^{n-d} L_{t+d+4}\right)\left(\prod_{t=1}^{n} F_{t+d+10}\right)} .
\end{aligned}
$$

Theorem 3.8. For $1 \leq d \leq n$,

$$
\begin{aligned}
U_{d, n}^{-1} & =(-1)^{d+n(k-1)} \mathbf{i}^{k(k+r+s+1)} q^{\left(\begin{array}{c}
d-1 \\
2
\end{array}\right)+\left(\begin{array}{l}
n \\
2
\end{array}\right)-\frac{k(k+r+s+1)}{2}+s} \\
& \times\left(1-q^{2 d+r-s}\right) \frac{1}{(q ; q)_{n-d}(q ; q)_{d-1}} \\
& \times \frac{\left(-q^{d+r+2} ; q\right)_{n+k-2}\left(-q^{-d+s+2} ; q\right)_{n+k-2}\left(-q^{n+k+r+1} ; q\right)_{n}\left(-q^{-n-k-s+1} ; q\right)_{n}}{\left(q^{k} ; q\right)_{n-1}\left(q^{d+r-s+1} ; q\right)_{n}\left(q^{n+k+r+s+2} ; q\right)_{n-1}} .
\end{aligned}
$$

Fibonacci-Lucas corollary for $k=3, r=4$, and $s=2$ :

Corollary 3.9. For $1 \leq d \leq n$,

$$
\begin{aligned}
U_{d, n}^{-1} & =(-1)^{\left(\begin{array}{c}
d \\
2
\end{array}\right)+\left(\begin{array}{l}
n \\
2
\end{array}\right)+1} F_{2(d+1)} \\
\times & \frac{\left(\prod_{t=1}^{n+1} L_{t+d+5}\right)\left(\prod_{t=1}^{n+1} L_{t-d+3}\right)\left(\prod_{t=1}^{n} L_{t+n+7}\right)\left(\prod_{t=1}^{n} L_{t-n-5}\right)}{\left(\prod_{t=1}^{n-1} F_{t+2}\right)\left(\prod_{t=1}^{n-d} F_{t}\right)\left(\prod_{t=1}^{d-1} F_{t}\right)\left(\prod_{t=1}^{n} F_{t+d+2}\right)\left(\prod_{t=1}^{n-1} F_{t+n+10}\right)} .
\end{aligned}
$$

Now we consider the inverse matrix. Since $\mathcal{B}^{-1}=U^{-1} L^{-1}$ and by the definitions of the matrices $U^{-1}$ and $L^{-1}$, we have the following result without proof. 
Theorem 3.10. For $n>0$,

$$
\begin{aligned}
& \left(\left(\mathcal{B}_{n}\right)^{-1}\right)_{i, j}=(-1)^{-j-j k+i} \mathbf{i}^{k(k+r+s+1)} \\
& \times q^{\left(\begin{array}{c}
(j+1 \\
2
\end{array}\right)-j k+s-\frac{1}{2} k(r+s+k)-\frac{1}{2}(3 i+k-1)} \frac{\left(1-q^{2 i+r-s}\right)\left(1-q^{2 j+r+s+k+1}\right)}{(q ; q)_{i-1}(q ; q)_{j-1}} \\
& \times \sum_{t} q^{t(t+k-j-1)} \frac{\left(-q^{i+r+2} ; q\right)_{t+k-2}\left(-q^{-i+s+2} ; q\right)_{t+k-2}\left(-q^{t+k+r+1} ; q\right)_{t}}{\left(q^{k} ; q\right)_{t-1}(q ; q)_{t-i}\left(q^{i+r-s+1} ; q\right)_{t}\left(q^{t+k+r+s+2} ; q\right)_{t-1}} \\
& \times \frac{(q ; q)_{t-1}\left(-q^{j+r+2} ; q\right)_{t-j}\left(-q^{j-t+s+2} ; q\right)_{t-j}\left(q^{t+k+r+s+2} ; q\right)_{t-1}\left(-q^{-t-k-s+1} ; q\right)_{t}}{(q ; q)_{t-j}\left(-q^{t+j+k+r} ; q\right)_{t-j}\left(-q^{j+k+s} ; q\right)_{t-j}\left(q^{j+k+r+s+2} ; q\right)_{t}} .
\end{aligned}
$$

The final formula as given in the theorem just above follows from some straightforward simplifications. Unfortunately, the sum again cannot be evaluated in closed form as we saw.

\section{Proofs}

Here we shall only prove four claimed identities related with matrices $\mathcal{A}$ and $\mathcal{B}$ in the following subsections separately. We use the $q$-Zeilberger algorithm for all the identities will be proven, our experiments indicate that they are Gosper-summable. The entries in our examples, qualify for the $q$-Zeilberger algorithm that we used in our earlier papers. Nowadays, such identities are a routine verification using the $q$-Zeilberger algorithm, as described in the book [18].

\subsection{Proofs related with the matrix $\mathcal{A}$}

We shall present the proofs related to the matrix $\mathcal{A}$. For $L U$-decomposition of $\mathcal{A}$, we have to prove that

Thus, consider

$$
\sum_{1 \leq t \leq \min \{d, n\}} L_{d, t} U_{t, n}=\mathcal{A}_{d, n}
$$

$$
\begin{aligned}
& \sum_{1 \leq t \leq \min \{d, n\}} L_{d, t} U_{t, n} \\
= & (-1)^{k(d-1)} \mathbf{i}^{k(k-r-s)} q^{k(d+1)+n+\frac{k(k+r+s)}{2}-1}(1-q)^{k}(q ; q)_{d-1}(q ; q)_{n-1} \\
\times & \sum_{1 \leq t \leq \min \{d, n\}} q^{t(1-n-k)} \frac{1}{(q ; q)_{n-t}(q ; q)_{d-t}} \\
\times & \frac{\left(q^{k} ; q\right)_{t-1}\left(-q^{2 t+k+s+r+1} ; q\right)_{d-t}\left(-q^{t+k+r+s+2} ; q\right)_{t-1}\left(-q^{n+r-s+1} ; q\right)_{t-1}}{(q ; q)_{t-1}\left(-q^{t+k+s+r+2} ; q\right)_{d-t}\left(-q^{-t-k-s+1} ; q\right)_{t-1}\left(q^{t+k+r+1} ; q\right)_{t-1}} \\
\times & \frac{\left(q^{t+r+2} ; q\right)_{t+k-1}\left(-q^{s+1} ; q\right)_{t+k-1}}{\left(q^{d+r+2} ; q\right)_{t+k-1}\left(-q^{d-t+s+1} ; q\right)_{t+k-1}\left(q^{n+r+2} ; q\right)_{t+k-1}\left(-q^{s-n+2} ; q\right)_{t+k-1}}
\end{aligned} .
$$

Denote the last sum in the above equation by $\operatorname{SuM}_{n}$. The Mathematica version of the $q$-Zeilberger algorithm produces the recursion

$$
\operatorname{SUM}_{n}=\frac{\left(1-q^{d+n+r}\right)\left(1+q^{d-n+k+s+1}\right)}{q\left(1-q^{n-1}\right)\left(1-q^{d+n+k+r}\right)\left(1+q^{d-n+s+1}\right)} \operatorname{SUM}_{n-1} .
$$

Since

$$
\mathrm{SUM}_{1}=\frac{q^{s}}{(q ; q)_{d-1}\left(q^{d+r+2} ; q\right)_{k}\left(-q^{d+s} ; q\right)_{k}},
$$


we obtain

$$
\operatorname{SUM}_{n}=\frac{1}{q^{n-1-s}(q ; q)_{d-1}\left(q^{2} ; q^{2}\right)_{k}(q ; q)_{n-1}}\left[\begin{array}{c}
n+d+k+r \\
n+d+r
\end{array}\right]_{q}^{-1}\left[\begin{array}{c}
d-n+k+s \\
d-n+s
\end{array}\right]_{-q}^{-1},
$$

as claimed.

Now we prove

$$
\sum_{d \leq t \leq n} L_{n, t} L_{t, d}^{-1}=\delta_{n, d}
$$

where $\delta_{n, d}$ is the Kronecker delta. By lower triangular matrices $L$ and $L^{-1}$, we need to look at the entries indexed by $(n, d)$ :

$$
\begin{aligned}
& \sum_{d \leq t \leq n} L_{n, t} L_{t, d}^{-1}=(-1)^{-d+d k+k n} q_{2}^{\left(\begin{array}{c}
d+1 \\
2
\end{array}\right)}\left(1+q^{2 d+k+r+s+1}\right) \frac{(q ; q)_{n-1}}{(q ; q)_{d-1}} \\
& \times \sum_{d \leq t \leq n}(-1)^{t} \frac{q^{\left(\begin{array}{l}
t \\
2
\end{array}\right)-d t}\left(-q^{s+1} ; q\right)_{t+k-1}\left(-q^{2 t+k+s+r+1} ; q\right)_{n-t}}{\left(q^{n+r+2} ; q\right)_{t+k-1}\left(-q^{n-t+s+1} ; q\right)_{t+k-1}\left(-q^{t+k+s+r+2} ; q\right)_{n-t}} \\
& \times \frac{\left(q^{t+r+2} ; q\right)_{t+k-1}\left(q^{d+r+2} ; q\right)_{t-d}\left(-q^{d-t+s+2} ; q\right)_{t-d}\left(-q^{t+k+s+r+2} ; q\right)_{t-1}}{(q ; q)_{n-t}(q ; q)_{t-d}\left(q^{t+d+k+r} ; q\right)_{t-d}\left(-q^{d+k+s} ; q\right)_{t-d}\left(-q^{d+k+s+r+2} ; q\right)_{t}} .
\end{aligned}
$$

For the sum in the last expression, that is,

$$
\begin{aligned}
& \sum_{d \leq t \leq n}(-1)^{t} \frac{q^{\left(\begin{array}{l}
t \\
2
\end{array}\right)-d t}\left(-q^{s+1} ; q\right)_{t+k-1}\left(-q^{2 t+k+s+r+1} ; q\right)_{n-t}}{\left(q^{n+r+2} ; q\right)_{t+k-1}\left(-q^{n-t+s+1} ; q\right)_{t+k-1}\left(-q^{t+k+s+r+2} ; q\right)_{n-t}} \\
& \times \frac{\left(q^{t+r+2} ; q\right)_{t+k-1}\left(q^{d+r+2} ; q\right)_{t-d}\left(-q^{d-t+s+2} ; q\right)_{t-d}\left(-q^{t+k+s+r+2} ; q\right)_{t-1}}{(q ; q)_{n-t}(q ; q)_{t-d}\left(q^{t+d+k+r} ; q\right)_{t-d}\left(-q^{d+k+s} ; q\right)_{t-d}\left(-q^{d+k+s+r+2} ; q\right)_{t}} .
\end{aligned}
$$

the $q$-Zeilberger algorithm evaluates it as 0 provided that $i \neq j$. If $i=j$, it obvious that the sum is equal to 1 . Thus

$$
\sum_{d \leq t \leq n} L_{n, t} L_{t, d}^{-1}=\delta_{n, d}
$$

as claimed.

Similarly, using the $q$-Zeilberger algorithm, one could prove the result

$$
\sum_{d \leq t \leq n} U_{d, t} U_{t, n}^{-1}=\delta_{d, n}
$$

\subsection{Proofs related with the matrix $\mathcal{B}$}

In this part we shall give the proofs related to the matrix $\mathcal{B}$. For $L U$-decomposition of $\mathcal{B}$, we have to prove that

$$
\sum_{1 \leq t \leq \min \{d, n\}} L_{d, t} U_{t, n}=\mathcal{B}_{d, n}
$$


Thus, consider

$$
\begin{aligned}
& \sum_{1 \leq t \leq \min \{d, n\}} L_{d, t} U_{t, n} \\
= & (-1)^{k(d-1)} \mathbf{i}^{k(k-r-s-1)} q^{\left(\begin{array}{c}
k+1 \\
2
\end{array}\right)+\frac{1}{2} k(r+s+2 n)+k+n-1} \\
\times & (q ; q)_{n-1} \sum_{1 \leq t \leq \min \{d, n\}} q^{t(1-k-n)} \frac{\left(q^{d-t+1} ; q\right)_{t-1}}{(q ; q)_{t-1}(q ; q)_{n-t}} \\
\times & \frac{\left(-q^{t+r+2} ; q\right)_{t+k-1}\left(-q^{s+1} ; q\right)_{t+k-1}}{\left(-q^{d+r+2} ; q\right)_{t+k-1}\left(-q^{d-t+s+1} ; q\right)_{t+k-1}\left(q^{t+k+r+s+2} ; q\right)_{t-1}} \\
\times & \frac{\left(q^{k} ; q\right)_{t-1}\left(q^{n+r-s+1} ; q\right)_{t-1}\left(q^{t+k+r+s+2} ; q\right)_{t-1}\left(q^{d+k+r+s+2} ; q\right)_{t-1}}{\left(-q^{t+k+r+1} ; q\right)_{t-1}\left(-q^{n+r+2} ; q\right)_{t+k-1}\left(-q^{-n+s+2} ; q\right)_{t+k-1}\left(-q^{-t-k-s+1} ; q\right)_{t-1}} .
\end{aligned}
$$

Denote the last sum in the above equation by $\mathrm{SUM}_{n}$. The algorithm produces the recursion

$$
\operatorname{SUM}_{n}=\frac{q^{-1}\left(1+q^{d+n+r}\right)\left(1+q^{d-n+k+s+1}\right)}{\left(1-q^{n-1}\right)\left(1+q^{d+n+k+r}\right)\left(1+q^{d-n+s+1}\right)} \operatorname{SUM}_{n-1}
$$

Since

we obtain

$$
\mathrm{SUM}_{1}=\frac{q^{s}}{\left(-q^{d+k+r+1} ; q^{-1}\right)_{k}\left(-q^{d+s} ; q\right)_{k}},
$$

$$
\operatorname{SUM}_{n}=\frac{q^{s-n+1}}{(-q ; q)_{k}(-q ; q)_{k}(q ; q)_{n-1}}\left[\begin{array}{c}
n+d+k+r \\
n+d+r
\end{array}\right]_{-q}^{-1}\left[\begin{array}{c}
d-n+k+s \\
d-n+s
\end{array}\right]_{-q}^{-1}
$$

as claimed.

We continue with proving

$$
\sum_{d \leq t \leq n} U_{d, t} U_{t, n}^{-1}=\delta_{n, d}
$$

where $\delta_{n, d}$ is the Kronecker delta. By the lower triangular matrices $U$ and $U^{-1}$, we need to look at the entries indexed by $(n, d)$ :

$$
\begin{aligned}
& \sum_{d \leq t \leq n} U_{d, t} U_{t, n}^{-1}=(-1)^{-n+k(d+n)} q^{\left(\begin{array}{c}
n \\
2
\end{array}\right)+d+k+s} \\
& \times \frac{\left(q^{k} ; q\right)_{d-1}\left(-q^{n+k+r+1} ; q\right)_{n}\left(-q^{-n-k-s+1} ; q\right)_{n}\left(q^{d+k+r+s+2} ; q\right)_{d-1}}{\left(q^{k} ; q\right)_{n-1}\left(-q^{-d-k-s+1} ; q\right)_{d-1}\left(q^{n+k+r+s+2} ; q\right)_{n-1}\left(-q^{d+k+r+1} ; q\right)_{d-1}} \\
& \times \sum_{d \leq t \leq n}(-1)^{t} q^{\left(\begin{array}{l}
t \\
2
\end{array}\right)-d t} \frac{\left(1-q^{2 t+r-s}\right)}{(q ; q)_{t-d}(q ; q)_{n-t}} \\
& \times \frac{\left(q^{t+r-s+1} ; q\right)_{d-1}\left(-q^{t+r+2} ; q\right)_{n+k-2}\left(-q^{-t+s+2} ; q\right)_{n+k-2}}{\left(-q^{t+r+2} ; q\right)_{d+k-1}\left(-q^{-t+s+2} ; q\right)_{d+k-1}\left(q^{t+r-s+1} ; q\right)_{n}} .
\end{aligned}
$$

For the sum in the last expression, that is,

$$
\begin{aligned}
& \sum_{d \leq t \leq n}(-1)^{t} q^{\left(\begin{array}{l}
t \\
2
\end{array}\right)-d t} \frac{\left(1-q^{2 t+r-s}\right)}{(q ; q)_{t-d}(q ; q)_{n-t}} \\
& \times \frac{\left(q^{t+r-s+1} ; q\right)_{d-1}\left(-q^{t+r+2} ; q\right)_{n+k-2}\left(-q^{-t+s+2} ; q\right)_{n+k-2}}{\left(-q^{t+r+2} ; q\right)_{d+k-1}\left(-q^{-t+s+2} ; q\right)_{d+k-1}\left(q^{t+r-s+1} ; q\right)_{n}}
\end{aligned}
$$


the $q$-Zeilberger algorithm evaluates it as 0 for $i \neq j$. If $i=j$, it is obvious that the sum is equal to 1 . Thus

$$
\sum_{d \leq t \leq n} U_{d, t} U_{t, n}^{-1}=\delta_{d, n}
$$

as claimed. Similarly, using the $q$-Zeilberger algorithm, we have

$$
\sum_{d \leq t \leq n} L_{n, t} L_{t, d}^{-1}=\delta_{n, d}
$$

\section{References}

[1] C. Berg, Fibonacci numbers and orthogonal polynomials, Arab. J. Math. Sci. 17, 7588, 2011.

[2] L. Carlitz, Some determinants of q-binomial coefficients, J. Reine Angew. Math. 226, 216-220, 1967.

[3] W. Chu, On the evaluation of some determinants with q-binomial coefficients, J. Systems Sci. Math. Science 8 (4), 361-366, 1988.

[4] W. Chu, Generalizations of the Cauchy determinant, Publ. Math. Debrecen 58 (3), 353-365, 2001.

[5] W. Chu and L. Di Claudio, Binomial determinant evaluations, Ann. Comb. 9 (4), 363-377, 2005.

[6] W. Chu, Finite differences and determinant identities, Linear Algebra Appl. 430, 215-228, 2009.

[7] M.E.H. Ismail, One parameter generalizations of the Fibonacci and Lucas numbers, The Fibonacci Quart. 46/47, 167-180, 2008/2009.

[8] E. Kılıç and H. Prodinger, A generalized Filbert matrix, The Fibonacci Quart. 48, 29-33, 2010.

[9] E. Kılıç and H. Prodinger, The q-Pilbert matrix, Int. J. Comput. Math. 89, 13701377, 2012.

[10] E. Kılıç and H. Prodinger, Variants of the Filbert matrix, The Fibonacci Quart. 51, 153-162, 2013.

[11] E. Kılıç and H. Prodinger, Asymmetric generalizations of the Filbert matrix and variants, Publ. Inst. Math. (Belgrad) (N.S) 95 (109), 267-280, 2014.

[12] E. Kılıç and H. Prodinger, The generalized q-Pilbert matrix, Math. Slovaca 64, 10831092, 2014.

[13] E. Kılıç and H. Prodinger, The generalized Lilbert matrix, Periodica Math. Hungar. 73, 62-72, 2016.

[14] G.Y. Lee, S.G. Lee, and H.G. Shin, On the k-generalized Fibonacci matrix $Q_{K}^{*}$, Linear Algebra Appl. 251, 73-88, 1997.

[15] G.Y. Lee and S.H. Cho, The generalized Pascal matrix via the generalized Fibonacci matrix and the generalized Pell matrix, J. Korean Math. Soc. 45 (2), 479-491, 2008.

[16] M. Merca, A note on the determinant of a Toeplitz-Hessenberg matrix, Spec. Matrices 1, 10-16, 2013.

[17] A.M. Ostrowski, On some determinants with combinatorial numbers, J. Reine Angew. Math. 216, 25-30, 1964.

[18] M. Petkovsek, H. Wilf, and D. Zeilberger, $A=B$, A.K. Peters, Wellesley, MA, 1996.

[19] H. Prodinger, A generalization of a Filbert matrix with 3 additional parameters, Trans. Roy. Soc. South Afr. 65, 169-172, 2010.

[20] T.M. Richardson, The Filbert matrix, The Fibonacci Quart. 39 (3), 268-275, 2001.

[21] J. Zhou and J. Zhaolin, The spectral norms of g-circulant matrices with classical Fibonacci and Lucas numbers entries, Appl. Math. Comput. 233, 582-587, 2014.

[22] J. Zhou and J. Zhaolin, Spectral norms of circulant-type matrices with binomial coefficients and Harmonic numbers, Int. J. Comput. Math. 11 (5), 1350076, 2014. 